\title{
Characterization of biological liquids by Modulated 3D Cross- Correlation Dynamic Light Scattering
}

\author{
Eleonora Frau and Silvia Schintke \\ Laboratory of Applied NanoSciences (COMATEC-LANS), Department of Industrial Technologies, HEIG-VD \\ HES-SO // University of Applied Sciences Western Switzerland, CH-1401 Yverdon-les-Bains, Switzerland \\ e-mail: eleonora.frau@heig-vd.ch,silvia.schintke@heig-vd.ch
}

\begin{abstract}
The deep biomedical knowledge reached in the past decades in many fields of medicine and biology has been accompanied by continuous technological progress in measurement instrumentation and analysis techniques. This development is important also to improve the characterization and the understanding of the interactions between nanoparticles and biological liquids, due to emerging applications such as drug delivery, bioimaging, biosensing, diagnostics and photothermal therapy.

In this study, we characterize the interaction between mouse serum and gold nanoparticles (NPs) and nanorods (NRs) by Modulated 3D Cross-correlation Dynamic Light Scattering (DLS), in order to demonstrate that this technique can be applied to the investigation of complex biological liquids. The analysis of the size distribution of the hydrodynamic radius reveals three different contributions from particles motion, associated to rotation, translation and agglomerates. Moreover, we show that the interaction between Au NPs or NRs and mouse serum depends on the aspect ratio of the Au particles. These results are promising for deepening the knowledge on proteins-nanoparticles interaction, for laboratory-based experiments as well as for sensing and diagnostic applications and nanoparticles based medical therapy.
\end{abstract}

Keywords: dynamic light scattering, gold nanorods, gold nanoparticles, mouse serum, protein interaction, biomedical.

\section{INTRODUCTION}

In the biomedical field, it is important to use characterization methods that are not invasive and non-destructive. Modulated 3D Cross-Correlation Dynamic Light Scattering (DLS) is a recent non-destructive technique often used for nano- and microparticle size determination [1], within a range of $0.1 \mathrm{~nm}$ to $10 \mu \mathrm{m}$, even in turbid suspensions $[2,3]$. Due to these capabilities, in the last years DLS has been increasingly used also for the characterization of biological samples, such as proteins solutions $[4,5]$.

Gold nanoparticles (Au NPs) and nanorods (NRs) are already widely studied by DLS [6,7] in order to monitor their size and homogeneity after synthesis processes. It has been shown that with this technique it is possible to analyse the contribution to the motion due to rotation of non-spherical particles [7], and that the obtained information can be used to determine the degree of the non-sphericity of particles. Along with the increasing importance of Au NPs in biomedical applications, i.e. drug delivery, bioimaging, biosensing and photothermal therapy $[8,9]$, DLS is becoming a powerful method to study NPs interaction with proteins. To our knowledge, so far, well defined protein solutions have been taken into account for DLS studies, e.g. [4,7].

In this research, we focus our attention on the interaction between Au NPs and Au NRs of different dimensions with mouse serum. We demonstrate the successful application of DLS for determining the size distribution of components in such a complex system, and we show how the calculated hydrodynamic radii can be used to analyse the shape of agglomerates and the interaction between particles.

\section{EXPERIMENTAL}

\subsection{Materials}

Four different aqueous suspensions of gold NPs and NRs, all stabilized by bare citrate (purchased from NanoComposix), were used in the present work:

- Spherical NPs, with nominal diameter of 40nm (Au NPs 40nm), concentration of $0.05 \mathrm{mg} / \mathrm{mL}$;

- NRs with absorption peak at $660 \mathrm{~nm}$, nominal length and diameter of $48 \mathrm{~nm}$ and $18 \mathrm{~nm}$ respectively, aspect ratio of 2.6 (Au NRs AR2.6), concentration of $31 \mu \mathrm{g} / \mathrm{mL}$;

- NRs with absorption peak at 800nm, nominal length and diameter of $55 \mathrm{~nm}$ and $15 \mathrm{~nm}$ respectively, aspect ratio of 3.6 (Au NRs AR3.6), concentration of $16 \mu \mathrm{g} / \mathrm{mL}$;

- NRs with absorption peak at $980 \mathrm{~nm}$, nominal length and diameter of $85 \mathrm{~nm}$ and $15 \mathrm{~nm}$ respectively, aspect ratio of 5.6 (Au NRs AR5.6), concentration of $18 \mu \mathrm{g} / \mathrm{mL}$.

Mouse serum was purchased by BioWest, already sterile and filtered. In order to increase the scattering intensity in the DLS and to decrease the presence of large agglomerates additional treatments were performed. The mouse serum has been filtered through a membrane with pores of $1.2 \mu \mathrm{m}$ diameter. Samples of different concentrations $(33 \%, 44 \%, 50 \%$ and $60 \%)$ were prepared by dilution in ultra-pure DNase-free distilled water, followed by 
centrifugation at $4000 \mathrm{rpm}$ for 2 hours. The interaction of Au NPs and NRs with mouse serum was studied by adding $7 \%$ of NPs suspension ( $50 \mu \mathrm{L}$ in $700 \mu \mathrm{L}$ of serum). Glass cuvettes of $10 \mathrm{~mm} \times 10 \mathrm{~mm}$ were used for DLS.

\subsection{Modulated 3D cross-correlation dynamic light scattering}

NP sizing by DLS is based on Rayleigh scattering, i.e. particles are supposed to be significantly smaller than laser wavelength, and on Brownian motion. The scattered intensity is measured and analysed; it decays exponentially, and its decay parameter is proportional to the translational diffusion coefficient, $\mathrm{D}_{\mathrm{t}}$. For spherical particles, it can be calculated by the Stokes-Einstein equation:

$$
D_{t}=\frac{k T}{6 \pi \eta R_{H}}
$$

where $k$ is the Boltzmann constant, $T$ is the temperature, $\eta$ is the viscosity and $R_{H}$ the hydrodynamic radius.

Our system is equipped with an innovative setup (LS Instruments) that uses two laser beams, and two detectors, temporally isolated from each other, with the suppression of signal cross talk. Thanks to this configuration, we can measure even turbid samples, because multiple scattering is significantly reduced. The laser has a wavelength of $685 \mathrm{~nm}$, the high-speed intensity modulators have a frequency of $5 \mathrm{MHz}$ and the scattering angle is $90^{\circ}$.

All data reported in the present paper were acquired at a temperature of $20^{\circ} \mathrm{C}$. We have recorded three repetitions of 40 s per sample, and we have performed the analysis on the intensity size distribution by the CONTIN fit method [10], since our samples are mostly polydisperse. As a result, we show an average of the measured hydrodynamic radius with its standard deviation.

\subsection{UV-vis-NIR spectroscopy}

Optical absorbance spectra are recorded in the range from $200 \mathrm{~nm}$ and $1000 \mathrm{~nm}$ using an UV-vis-NIR spectrometer (OceanOptics Flame, cuvettes with $5 \mathrm{~mm}$ path). Both deuterium and halogen lamp were used.

\section{RESULTS AND DISCUSSION}

\subsection{Au NPs and mouse serum characterization}

The optical properties of gold NPs and NRs have been characterized by absorbance spectroscopy. In Fig.1(a) a comparison of the different spectra is shown. For the Au NRs we can observe the two surface plasmon resonance (SPR) bands: the transversal one is around $510 \mathrm{~nm}$, while the longitudinal one varies with the aspect ratio of the nanorods [11]. On the other hand, for Au NPs $40 \mathrm{~nm}$, only the transversal band is present. The absorbance peaks are in agreement with the values reported by the supplier.
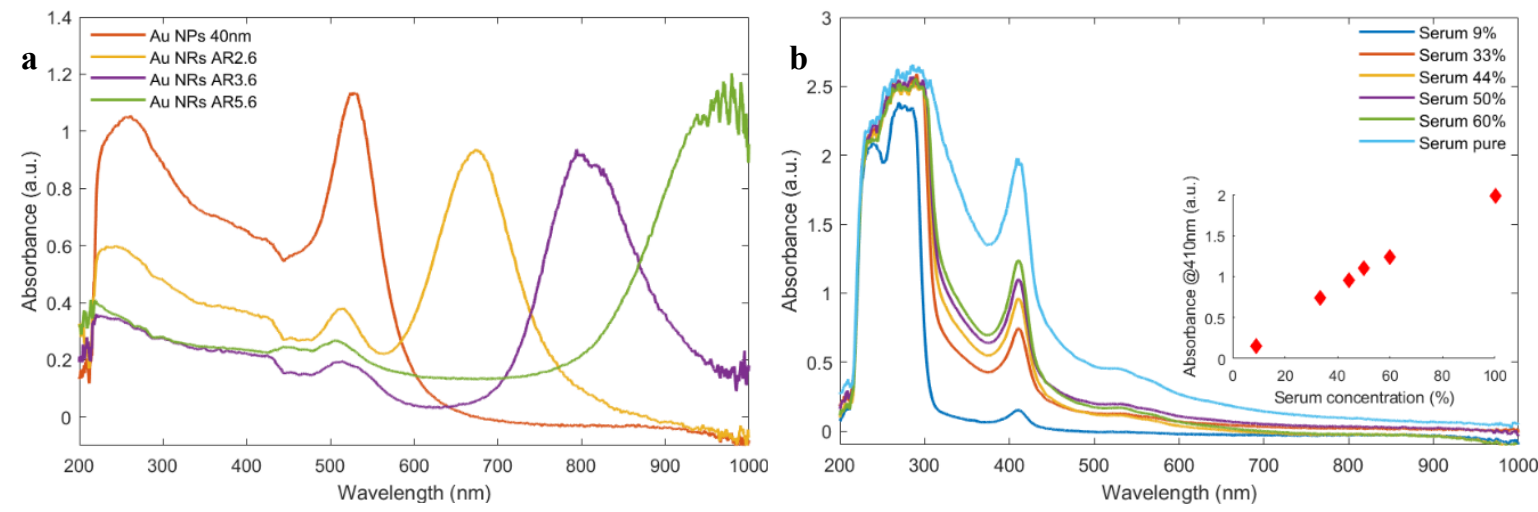

Figure 1. (a) Absorbance spectra of gold NPs and NRs. (b) Absorbance spectra of filtered mouse serum for different concentrations; the inset shows the essentially linear increase of the absorbance peak at $410 \mathrm{~nm}$.

Mouse serum was also studied by absorbance spectroscopy, as shown in Fig.1(b). From the spectra, the absorbance peak in the ultraviolet range, a characteristic of proteins, is clearly visible, together with a peak at 410 $\mathrm{nm}$. We do believe that this peak is related to enzymatic reactions, or to other components of mouse serum, and indeed it varies linearly with the concentration, as shown in the inset in Fig.1(b). For the following discussion we will mention proteins as the interactive components of mouse serum, although its composition is more differentiated.

DLS measurements were performed firstly on Au NPs and NRs suspensions, as shown in Fig.2(a). For Au NPs $40 \mathrm{~nm}$, only the peak related to translational diffusion is present, and it has an average value of $R_{H}=27.6 \mathrm{~nm}$. It is well known that the hydrodynamic radius of particles in liquids is larger than the one of dried particles [12]; this can be explained by the formation of a corona around the particles, due to the interactions with the molecules within the liquid. The size distribution of Au NRs shows always two peaks (Fig.2(a)). One is related to translational diffusion, corresponding to a hydrodynamic radius of around $19 \mathrm{~nm}$, and another is correlated to rotational motion, 
corresponding to $2 \mathrm{~nm}$ radius. This is in agreement with earlier observations by DLS on non-spherical particles [7]. In spite of similar detections of rotational modes, to our knowledge it is still hard to identify the nanorods dimensions, i.e. diameter and length, by a standard DLS experiment. However, our data allow us to make some considerations about their shape. In fact, we observe that the standard deviation of the translational peak increases by increasing the aspect ratio; i.e. longer nanorods result in a broader distribution of the hydrodynamic radius, which can be related to translational motion (Fig.2(a)).

Samples of mouse serum at different concentrations were also analysed by DLS, the results are shown in Fig.2(b). The sample with $9 \%$ dilution was not enough concentrated for reliable DLS data, so we don't take it into account for the further analysis. A common feature of the DLS measurements on mouse serum is the presence of three different hydrodynamic radii in the size distribution:

- a small one, between $2 \mathrm{~nm}$ and $5 \mathrm{~nm}$, which we associate to the rotational motion of proteins, and its scattered intensity can be an indication of the length of particles [7];

- a medium one, between $10 \mathrm{~nm}$ and $20 \mathrm{~nm}$, is related to the translational motion, so it is the value conventionally measured by a DLS acquisition;

- a large one, above $100 \mathrm{~nm}$ corresponds to the hydrodynamic radius of agglomerates present in the sample, where the rotation and translation contributions are not distinguished anymore [6].

We note that from the concentration of $60 \%$ the position of the translational peak starts to increase, which may indicate increasing protein-protein interaction. Moreover, in the case of non-diluted serum ( $100 \%$ concentration) the hydrodynamic radius of agglomerates is larger. Even if the values are still inside the error bar, this trend is confirmed by the value of the diffusion coefficient, which is reduced by almost $50 \%$ (from $7.8 \cdot 10^{-12} \pm 0.5 \cdot 10^{-12}$ $\mathrm{m}^{2} / \mathrm{s}$ for $33 \%$ concentration to $4.0 \cdot 10^{-12} \pm 0.4 \cdot 10^{-12} \mathrm{~m}^{2} / \mathrm{s}$ for $100 \%$ ). For these reasons, we have chosen a concentration of $33 \%$ for the following measurements. At this dilution, the hydrodynamic radius related to the translational peak is relatively small, providing the possibility to appreciate the interaction with added particles, such as NPs and NRs.
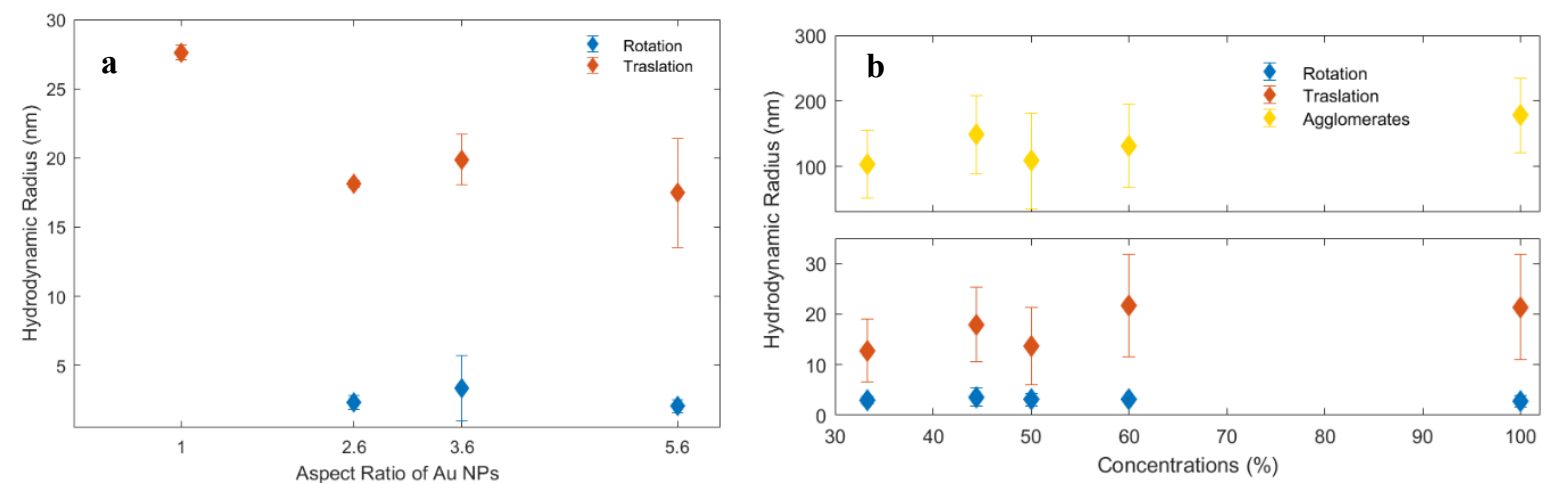

Figure 2. (a) Hydrodynamic radius of Au NPs and NRs as a function of their aspect ratio.

(b) Hydrodynamic radius of filtered mouse serum diluted at different concentrations.

\subsection{Interaction of Au NPs and mouse serum}

Results of the size distribution analysis from DLS spectra of filtered mouse serum diluted at 33\% and mixed with $7 \%$ of Au NPs and NRs are shown in Fig.3(a). The position labelled by "Serum 33\% pure" corresponds to the bare sample. In the presence of Au NPs $40 \mathrm{~nm}$ (aspect ratio 1) and Au NRs AR2.6 the size distributions don't present significant changes, suggesting that there is not a remarkable NP-protein interaction. To the contrary, Au NRs AR3.6 presents an increase of the translation peak by almost $10 \mathrm{~nm}$, and no agglomerates peak is present. A hypothesis that could explain this phenomenon is that the NRs-proteins interaction has as a consequence the formation of chains, and all the proteins get involved. Moreover, the big standard deviation of this peak indicates
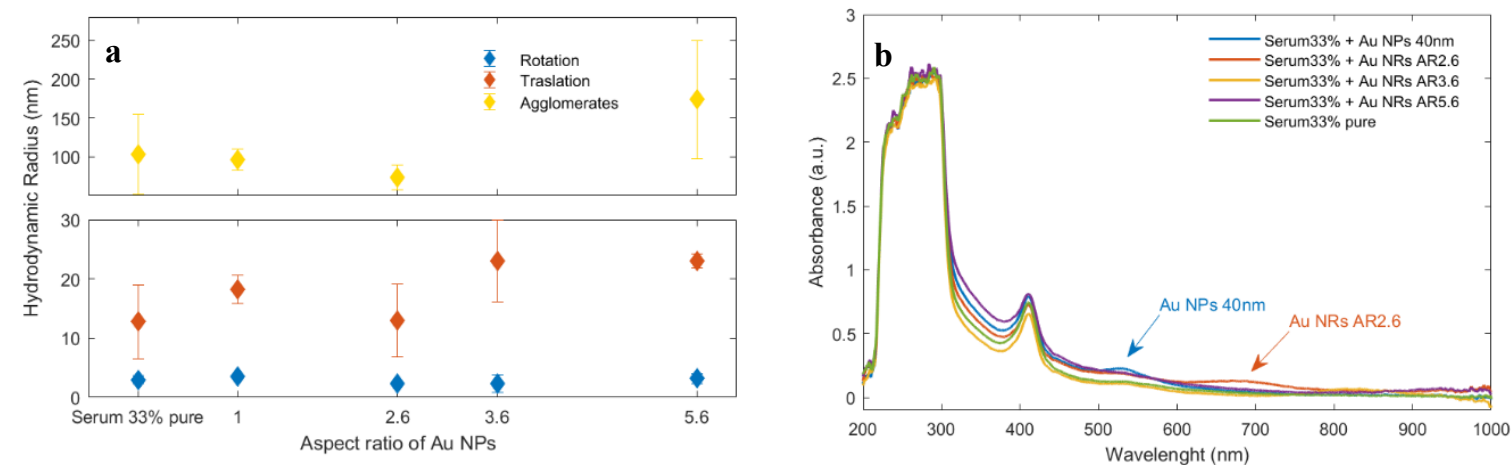

Figure 3. Hydrodynamic radius (a) and absorbance spectra (b) of mouse serum diluted at 33\% mixed with $A u$ NPs and NRs. 
that the length of these chains has a broad dispersion. Au NRs AR5.6 shows another interesting behaviour: the agglomerates peak has a value significantly larger than the others samples, with a large standard deviation, while the translational peak has a value comparable to Au NRs AR3.6 but with a smaller standard deviation (Fig.3(a)). These observations suggest that in this case proteins and NRs are interacting in such a way to form spherical conglomerations with large hydrodynamic radii. The Au NPs and NRs studied in this paper have the same surface stabilization, (citrate), therefor we suppose that its stability changes according to the aspect ratio of the particles, in line with previous observations of the interaction of monodisperse proteins with Au NRs [7,13].

The interactions between proteins and NRs could be further investigated, for example, by Atomic Force Microscopy (AFM), in order to elucidate the shape of agglomerates and to determine the different behaviour related to the NRs' aspect ratio.

To conclude the optical analysis of our samples, we performed absorbance spectroscopy on mouse serum with added Au NPs and NRs, as shown in Fig.3 (b). The position and the intensity of the absorbance peaks stays constant, and the peaks characteristic of Au SPR bands are slightly visible only for $\mathrm{Au} 40 \mathrm{~nm}$ and for Au NRs 2.6, as indicated by the arrows in the figure. We can conclude that, from an optical point of view, the addition of $\mathrm{Au}$ NPs and NRs to mouse serum samples doesn't bring any substantial changes to the UV-Vis-NIR absorbance spectra.

\section{CONCLUSIONS}

In this study we have investigated the interaction of mouse serum with Au NPs and NRs by DLS. We have shown that with this technique we can distinguish three different peaks in the hydrodynamic radius distribution, each one related to a different type of motion (i.e. rotation, translation, agglomerates). Furthermore, we have found that the NRs-proteins interaction depends on the aspect ratio. In particular, particles with aspect ratio of 1 and 2.6 don't influence the proteins size distribution, while particles with aspect ratios of 3.6 and 5.6 stimulate the formation of agglomerates of different shape. A similar behaviour has been reported for the study of various monodisperse proteins [7,13]. In our study, instead, we show that it is possible to evaluate the interaction of NPs and NRs even in a more complex biological fluid (mouse serum) thanks to the advantages of DLS.

\section{ACKNOWLEDGEMENTS}

This work has been financially supported by HEIG-VD and HES-SO, project PRONANO (grant number 81265/IA-EXT17-57).

\section{REFERENCES}

[1] S.K. Brar and M. Verma: Measurement of nanoparticles by light-scattering techniques, Trends in Analytical Chemistry, vol. 30, pp. 4-17, 2011.

[2] I.D. Block and F. Scheffold: Modulated 3D cross-correlation light scattering: Improving turbid sample characterization, Review of Scientific Instruments 81, 123107, 2010.

[3] E. Frau and S. Schintke: Modulated 3D Cross-Correlation Dynamic Light Scattering of Magnetic Nanoparticle Inks, Transparent Optical Networks (ICTON), 20th International Conference on, pp. 1-4, IEEE, 2018.

[4] J. Stetefeld at al.: Dynamic light scattering: a practical guide and applications in biomedical science, Biophys. Review, vol. 8, pp. 409-427, 2016.

[5] B. Lorber et al:: Protein analysis by dynamic light scattering: methods and techniques for students, Biochemistry and Molecular Biology Education, vol. 40, pp. 372-382, 2012.

[6] B.N. Khlebtsov and N.G. Khlebtsov: On the measurement of gold nanoparticles size by the dynamic light scattering method, Colloid Journal, vol. 73, pp. 118-127, 2011.

[7] H. Liu et al.: Dynamic light scattering for gold nanorod size characterization and study of nanorod-protein interactions, Gold Bull, Springer, vol. 45, pp. 187-195, 2012.

[8] E.C. Dreaden et al:: The golden age: gold nanoparticles for biomedicine, Chem. Soc. Rev., vol. 41, pp. 2740-2779, 2012.

[9] E. Boisselier, D. Astruc: Gold nanoparticles in nanomedicine: preparations, imaging, diagnostics, therapies and toxicity, Chem. Soc. Rev., vol. 38, pp. 1759-1782, 2009.

[10] S.W. Provencher: CONTIN: a general purpose constrained regularization program for inverting noisy linear algebraic and integral equations, Computer Physics Comm., vol. 27, pp. 229-242, 1982.

[11] S. Link et al.: Simulation of the optical absorption spectra of gold nanorods as a function of their aspect ratio and the effect of the medium dielectric constant, J. Phys Chem B, vol. 103, pp. 3073-3077, 1999.

[12] T. G. F. Souza et al.: A comparison of TEM and DLS methods to characterize size distribution of ceramic nanoparticles, J. Phys.: Conf. Ser., 733, 2016.

[13] B. Pan et al.: Study on interaction between gold nanorod and bovine serum albumin, Colloid Surf. A, vol. 295, pp. 217-222, 2007. 